\title{
Biometria ocular, erro refrativo e sua relação com a estatura, idade, sexo e escolaridade em adultos brasileiros
}

\author{
Ocularbiometry, refractive error and correlation with height, age, \\ genderand yearsofformal education
}

\author{
Graziela Campanelli Pereira ${ }^{1}$ \\ Norma Allemann²
}

Trabalho realizado na Universidade Federal de São Paulo - UNIFESP - São Paulo (SP) - Brasil.

${ }^{1}$ Pós-graduanda do Departamento de Oftalmologia da Universidade Federal de São Paulo - UNIFESP - São Paulo (SP) - Brasil.

${ }^{2}$ Doutora em Oftalmologia pela UNIFESP e Chefe do Setor de Ultra-som Ocular do Departamento de Oftalmologia da UNIFESP - São Paulo (SP) - Brasil.

Endereço para correspondência: Rua Pedro Pomponazzi, 40 - Apto. 32 - São Paulo (SP) CEP 04115000

E-mail: grazi10@hotmail.com

Recebido para publicação em 26.07.2006

Aprovado em 15.02.2007

\begin{tabular}{|l|}
\hline RESUMO \\
\hline Objetivo: Avaliar os parâmetros biométricos oculares e o erro refrativo em \\
adultos brasileiros e a sua relação com a estatura, idade, sexo e escolarida- \\
de. Métodos: Estudo transversal que avaliou 173 indivíduos pela cerato- \\
metria, ecobiometria, refração e aferição da estatura. A análise estatística \\
foi realizada pelo coeficiente de Pearson e pela elaboração de um modelo \\
de regressão. Resultados: Encontrou-se que: a cada aumento de 10 cm na \\
estatura, esperar-se-ia encontrar um comprimento axial0,32 mm mais longo, \\
profundidade da câmara anterior 0,07 mm mais profunda,profundidade da \\
cavidade vítrea 0,26 mm mais profunda e ceratometria 0,50 D mais plana; \\
a cada aumento de 10 anos na idade esperar-se-ia encontrar profundidade \\
da câmara anterior 0,15 mm mais rasa, cristalino 0,25 mm mais espesso, \\
profundidade da cavidade vítrea 0,21 mm mais rasa e equivalente esférico \\
0,23 D mais positivo; a cada 10 anos de escolaridade esperar-se-ia equiva- \\
lente esférico 0,74 D mais negativo. O sexo não apresentou influência. \\
Equações referentes aos parâmetros biométricos e ao equivalente esférico \\
foram formuladas. Conclusões: Correlações positivas foram encontradas \\
entre: comprimento axial e estatura; profundidade da câmara anterior e da \\
cavidade vítrea e estatura; espessura do cristalino e idade; ceratometria e \\
estatura; equivalente esférico e idade. Correlações negativas foram encon- \\
tradas entre: profundidade de câmara anterior e da cavidade vítrea e idade; \\
equivalente esférico e escolaridade.
\end{tabular}

Descritores: Olho; Biometria; Erros de refração; Estatura; Fatores etários; Fatores sexuais; Escolaridade

\section{INTRODUÇÃ̃O}

O olho humano é geneticamente programado para atingir a emetropia na juventude e mantê-la com o avançar da idade ${ }^{(1)}$, apesar de todas as alterações nos parâmetros biométricos oculares que podem ocorrer durante o período de crescimento e desenvolvimento do indivíduo.

Estudos sobre os componentes do crescimento ocular sugerem que o olho alcança o comprimento axial adulto de emetropia por volta dos 13 anos de idade $^{(2)}$ e um contínuo alongamento axial após essa idade é usualmente encontrado na miopia progressiva juvenil ${ }^{(3-4)}$.

O comprimento axial do globo ocular já foi relacionado a vários fatores: esforço acomodativo ${ }^{(5-6)}$, alterações biomecânicas ${ }^{(7)}$, estímulos bioquímicos locais ${ }^{(8)}$ e elementos anatômicos como a circunferência cefálica ${ }^{(9)}$ e a estatura dos indivíduos ${ }^{(10)}$. Estudos mencionam diferenças nas dimensões oculares entre indivíduos do sexo masculino e feminino ${ }^{(11-13)}$ e mudanças nos parâmetros biométricos com a escolaridade ${ }^{(3,6,14-15)}$. 
Embora a maioria dos pesquisadores concorde que o erro refrativo seja, em grande parte, geneticamente determinado, uma grande quantidade de evidências tem mostrado que experiências visuais precoces podem afetar o crescimento ocular e, no final, o estado refrativo ${ }^{(16)}$.

Considerando-se os achados descritos na literatura elaborou-se um protocolo de pesquisa que foi utilizado em indivíduos atendidos por demanda espontânea no Centro de Referência Secundário em Oftalmologia (CERESO), com o intuito de analisar, na população de adultos, parâmetros biométricos oculares e o erro refrativo associado à estatura, idade, sexo e escolaridade, fatores estes que hipoteticamente estariam relacionados ao crescimento ocular.

\section{MÉTODOS}

Este estudo transversal foi realizado na Universidade $\mathrm{Fe}$ deral de São Paulo - Escola Paulista de Medicina, Departamento de Oftalmologia, nos Setores de Ultra-som e no Centro de Referência Secundária em Oftalmologia (CERESO) na cidade de São Paulo, durante os anos de 2002 e 2003. Seu protocolo científico foi aprovado pelo Comitê de Ética Universitário (processo nำ770/01, 10/09/2001).

Após obtenção do consentimento livre permitido dos participantes, estes foram submetidos à entrevista e a exames, os dados foram compilados e ao paciente foi indicado o tratamento. Todos os exames foram realizados pelo mesmo examinador (autor).

Amostra: Neste estudo foram incluídos 173 indivíduos que procuraram espontaneamente o centro de referência com o objetivo de serem submetidos a uma consulta oftalmológica devido a diferentes causas.

Os critérios de inclusão foram: idade entre 21 e 70 anos; sem diagnóstico de diabetes mellitus ou de patologia ocular; sem antecedentes de cirurgia ocular. Foram excluídos os indivíduos que apresentaram, ao exame oftalmológico: acuidade visual corrigida inferior a 20/20; ambliopia; pressão intra-ocular acima de $21 \mathrm{mmHg}$ e/ou escavação do disco óptico maior que 0,5.

Procedimentos: Na realização da ceratometria e refratometria automatizadas utilizou-se o refrator e ceratômetro automático objetivo Topcon KR3000 (Paramus, NJ, EUA). A ceratometria foi realizada e para sua análise foi feita a média $(\mathrm{K})$ da ceratometria mais plana (K1) e mais curva (K2) de cada olho. Na realização da tonometria utilizou-se o auto-tonômetro de não contato (NT-2000, Nidek, Fremont, EUA) em ambos os olhos.

A informação sobre idade foi obtida através de pergunta direta ao paciente. Considerou-se a idade em anos (sem frações) que apresentava no dia do exame ${ }^{(17)}$.

A metodologia utilizada para a classificação dos indivíduos segundo a raça foi a mesma utilizada pelo IBGE ao realizar o Censo de $2000^{(17)}$. Os indivíduos foram questionados a classificarem-se em uma das raças: lia-se as possibilidades (branco, mulato e negro, amarelo e vermelho) e o indivíduo é quem escolhia, o que foi tido como verdadeiro.

A definição de escolaridade utilizada para este estudo foi o número de anos que o indivíduo estudou, independente do número de horas por dia dedicadas ao estudo e se houve sucesso ou não, na progressão da escolaridade. Dados sobre a escolaridade dos indivíduos foram obtidos pela pergunta "O (a) $\mathrm{Sr}$. (a) estudou? "Em caso de resposta positiva, era então perguntado: "Durante quantos anos? Até qual série? Repetiu algum ano?".

A mensuração da estatura do paciente foi realizada com o paciente em pé, sem sapatos, com a coluna ereta, de costas para a haste de mensuração da balança Filizola, modelo 31 (São Paulo, SP, Brasil) e os dados foram anotados em centímetros.

Os 346 olhos dos 173 indivíduos incluídos no protocolo foram submetidos à biometria ultra-sônica utilizando-se ultrasonografia modo A, transdutor de $10 \mathrm{MHz}$ Ultrascan ${ }^{\circledR}$, (Alcon, Irvine, EUA), técnica de contato na superfície da córnea.

As medidas das dimensões oculares dos olhos foram realizadas com o paciente deitado em decúbito dorsal horizontal, após a instilação de uma gota de colírio de cloridrato de proximetacaína a $0,5 \%$ (Anestalcon ${ }^{\circledR}$, Alcon Laboratórios do Brasil Ltda., São Paulo), no saco conjuntival inferior de ambos os olhos com o paciente deitado em posição supina. O biômetro foi utilizado sempre no modo "fácico", ganho de $80 \mathrm{~dB}$, congelamento automático dos ecogramas.

Solicitava-se que os olhos fossem mantidos em posição primária do olhar focalizando o teto da sala e as pálpebras foram contidas pelo examinador durante o exame. Alinhava-se a sonda do ultra-som com o objetivo de obter-se todos os ecos (córnea, cristalino e retina) os mais intensos possíveis e de intensidade semelhante ao eco retiniano, para assegurar que a medida estivesse sendo referente ao eixo axial ${ }^{(18)}$. Foram realizadas 10 medidas de cada olho e analisou-se o ecograma de cada aquisição e seu desvio padrão. Foram excluídos os ecogramas não adequados, sendo considerado necessário o mínimo de 5 medidas com bom alinhamento, apresentando desvio padrão máximo de $0,10 \mathrm{~mm}$.

A cicloplegia foi conseguida pela instilação de 1 gota do colírio de ciclopentolato a $1 \%$ (Cicloplégico ${ }^{\circledR}$, Allergan, São Paulo) e, após intervalo de 5 minutos, 1 gota do colírio de cloridrato de tropicamida a $1 \%$ (Mydriacyl ${ }^{\circledast}$, Allergan, São Paulo) em cada um dos dos olhos ${ }^{(19)}$.

A refratometria foi realizada entre 20 minutos e 1 hora da instilação da primeira gota de colírio. Foi realizada em quarto escuro, com optotipos projetados a uma distância de 4 metros. Somou-se ao componente esférico $0,25 \mathrm{D}$ como fator de correção da distância. Dados da refratometria foram analisados pelo equivalente esférico em dioptrias, baseando-se na refração subjetiva sob cicloplegia obtida de todos os indivíduos. O erro refrativo foi analisado pelo equivalente esférico (EE), calculado pela soma do componente esférico à metade do componente cilíndrico em dioptrias. Emetropia foi definida como EE entre $-0,50$ e $+0,50$ D. Miopia foi definida como $\mathrm{EE}<-0,50 \mathrm{D}$. Alta miopia foi definida como $E E<-5,00 \mathrm{D}$. Hipermetropia foi definida como EE $>+0,50 \mathrm{D}$. Anisometropia foi definida com a diferença de EE dos olhos direito e esquerdo maior que 1,00 D.

Utilizou-se o oftamoscópio indireto (Eyetec, modelo FOH 3.1-A) para a realização do exame da retina. 


\section{Definições e análise estatística}

Foram realizados os cálculos do coeficiente de correlação linear de Pearson entre os parâmetros biométricos e o equivalente esférico dos olhos direito e esquerdo de todos os indivíduos. Por terem sido encontrados valores indicando uma alta correlação dos parâmetros biométricos entre ambos os olhos de cada indivíduo, apenas os parâmetros referentes ao olho direito dos indivíduos foram utilizados nas análises.

Na condução da análise, inicialmente, foi calculado o coeficiente de correlação de Pearson para avaliar a associação entre cada uma das variáveis numéricas (duas a duas): comprimento axial (CAx); profundidade da câmara anterior (PCA); espessura do cristalino (EC); profundidade da cavidade vítrea $(\mathrm{PCV})$; ceratometria média $(\mathrm{K})$ e equivalente esférico $(\mathrm{EE})$.

Modelos de análise de regressão linear múltipla foram então construídos para avaliar o efeito da estatura, da idade, do sexo e da escolaridade (variáveis independentes) nos parâmetros biométricos individuais e na refração (variáveis dependentes).

A adequação dos ajustes foi avaliada pela construção de gráficos de resíduos dos modelos de regressão. Esses resíduos foram homogeneamente distribuídos no gráfico, sugerindo que estes modelos foram adequados.

Considerou-se estatisticamente significante se $\mathrm{p}<0,05$.

A correlação de Pearson foi graduada como: forte ( $r>0,70)$; moderada ( $\mathrm{r}=0,50$ a 0,70$)$; fraca $(\mathrm{r}=0,30 \mathrm{a}<0,50)$; e quando menor que 0,30 indicava que não havia associação entre as variáveis.

\section{RESULTADOS}

A amostra de 173 indivíduos apresentou maior porcentagem de indivíduos do sexo feminino (64,74\%). Em relação à raça, encontrou-se maior porcentagem de indivíduos da raça branca $(48,55 \%)$, seguida pela raça parda $(36,42 \%)$, negra $(13,30 \%)$ e vermelha $(1,73 \%)$. Não houve representante da raça amarela.

Quanto ao erro refrativo foram encontrados $22(12,72 \%)$ indivíduos míopes, 57 (32,95\%) emétropes; 94 (54,33\%) hipermétropes. Apenas $1(0,58 \%)$ indivíduo apresentava alta miopia e $11(6,36 \%)$ apresentavam anisometropia.

$\mathrm{Na}$ distribuição dos indivíduos quanto à idade, pôde-se observar que, com o aumento da idade, os indivíduos apresentaram tendência a maiores médias nas medidas: espessura do cristalino, ceratometria média e equivalente esférico do erro refrativo; e menores médias nas medidas: comprimento axial, profundidade da câmara anterior, profundidade da cavidade vítrea, estatura e escolaridade.

Em relação à estatura, pôde-se observar que os indivíduos com maior estatura apresentaram tendência a maiores médias nas medidas: comprimento axial, profundidade da câmara anterior, profundidade da cavidade vítrea e escolaridade; e menores médias da: espessura do cristalino, ceratometria média, equivalente esférico e idade.

A figura 1 mostra a distribuição dos indivíduos quanto à escolaridade.
Quando os indivíduos da amostra foram separados segundo o sexo, encontrou-se que os indivíduos do sexo masculino apresentaram tendência a maiores médias das medidas: comprimento axial, profundidade da câmara anterior, profundidade da cavidade vítrea, estatura e escolaridade. Já nos indivíduos do sexo feminino encontrou-se tendência a apresentarem maior média das medidas: espessura do cristalino, ceratometria média, equivalente esférico do erro refrativo e da idade.

A figura 2 mostra biometrias realizadas em indivíduos de idades, sexos e estaturas diferentes, e semelhante escolaridade e erro refrativo, mostrando os parâmetros biométricos analisados.

A tabela 1 mostra as correlações de Pearson encontradas entre os parâmetros biométricos, ceratometria média, equivalente esférico do erro refrativo, estatura, idade e escolaridade. Foi encontrada correlação forte $(r>0,70)$ entre: comprimento axial (CAx) e profundidade da cavidade vítrea (PCV). Correlação moderada ( $\mathrm{r}=0,50$ a 0,70$)$ positiva entre comprimento axial (CAx) e profundidade da câmara anterior (PCA); entre a espessura do cristalino (EC) e idade e negativa entre comprimento axial $(\mathrm{CAx})$ e a ceratometria média $(\mathrm{K})$; comprimento axial $(\mathrm{CAx})$ e equivalente esférico (EE); profundidade da câmara anterior (PCA) e a espessura do cristalino (EC); espessura do cristalino (EC) e profundidade da cavidade vítrea (PCV); profundidade da cavidade vítrea $(\mathrm{PCV})$ e a ceratometria média $(\mathrm{K})$; profundidade da cavidade vítrea (PCV) e equivalente esférico (EE). A correlação de Pearson foi considerada fraca $(r=0,30$ a $<0,50)$ e positiva entre comprimento axial (CAx) e estatura; profundidade da câmara anterior (PCA) e profundidade da cavidade vítrea (PCV); profundidade da cavidade vítrea (PCV) e estatura; e negativa entre comprimento axial (CAx) e espessura do cristalino (EC); profundidade da câmara anterior (PCA) e a idade; profundidade da cavidade vítrea (PCV) e idade; e entre a idade e a escolaridade

A tabela 2 mostra os resultados da aplicação de um modelo de regressão dos parâmetros biométricos oculares, ceratometria média e equivalente esférico do erro refrativo com a estatura, a idade e a escolaridade. De acordo com este modelo de regressão, é esperado que: uma pessoa $10 \mathrm{~cm}$ mais alta apresente o comprimento axial 0,32 mm mais longo, a câmara anterior $0,07 \mathrm{~mm}$ mais profunda, a cavidade vítrea $0,26 \mathrm{~mm}$ mais

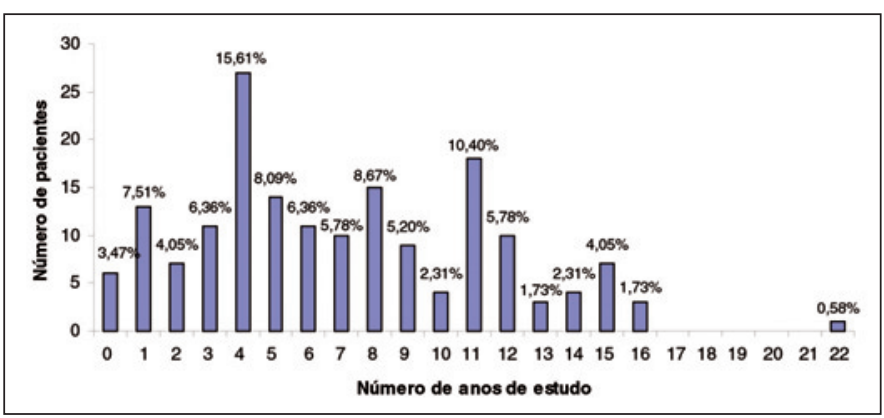

Figura 1 - Gráfico demonstrativo da distribuição dos indivíduos da amostra, referente à escolaridade (número de anos de estudo) 



Figura 2 - Ecogramas obtidos em exame de biometria ultra-sônica (modo A, técnica de contato) de pacientes demonstrando a profundidade de câmara anterior (AC); espessura do cristalino (L); profundidade da cavidade vítrea (V); e comprimento axial (AL): A) Idade de 23 anos: sexo masculino, estatura $171 \mathrm{~cm}$, escolaridade 7 anos, comprimento axial $24,30 \mathrm{~mm}$, profundidade da câmara anterior $4,21 \mathrm{~mm}$, espessura do cristalino $3,34 \mathrm{~mm}$, cavidade vítrea $16,75 \mathrm{~mm}$, equivalente esférico $+0,50 \mathrm{D}$. B) Idade de 60 anos: sexo feminino, estatura, $153 \mathrm{~cm}$, escolaridade 8 anos, profundidade da câmara anterior $2,88 \mathrm{~mm}$, espessura do cristalino $2,81 \mathrm{~mm}$, cavidade vítrea $14,10 \mathrm{~mm}$, equivalente esférico +050 $\mathrm{D}$.

Tabela 1. Valores das correlações de Pearson encontradas entre os parâmetros biométricos, ceratometria média, equivalente esférico do erro refrativo, estatura, idade e escolaridade



$\mathrm{CAx}=$ comprimento axial; $\mathrm{PCA}=$ profundidade da câmara anterior; $\mathrm{EC}=$ espessura do cristalino; $\mathrm{PCV}=$ profundidade da cavidade vítrea; $\mathrm{Km}=\mathrm{ceratometria} \mathrm{média;}$ $\mathrm{EE}=$ equivalente esférico

\begin{tabular}{|l|llllllllllll|}
\hline \multicolumn{2}{|c|}{ Tabela 2. Resultados da aplicação do modelo de regressão linear dos parâmetros biométricos oculares e do erro refrativo com estatura, } \\
idade, escolaridade e sexo
\end{tabular}

profunda e a ceratometria média (K) 0,50 D mais plana. Quanto à idade, é esperado que com 10 anos a mais, apresente a câmara anterior $0,15 \mathrm{~mm}$ mais rasa, o cristalino $0,25 \mathrm{~mm}$ mais espesso, a cavidade vítrea $0,21 \mathrm{~mm}$ mais rasa e o equivalente esférico do erro refrativo $0,23 \mathrm{D}$ mais positivo. Quanto à escolaridade, é esperado que uma pessoa com 10 anos a mais de estudo apresente equivalente esférico do erro refrativo $0,74 \mathrm{D}$ mais negativo.
Equações formuladas a partir dos coeficientes obtidos nos modelos de regressão:

$\mathrm{CAx}=18,192+0,032$ (estatura)

$\mathrm{PCA}=2,684+0,07$ (estatura) $-0,015$ (idade)

$\mathrm{EC}=3,083+0,025$ (idade)

$\mathrm{PCV}=12,316+0,026$ (estatura) $-0,021$ (idade)

$\mathrm{K}=52,090-0,050$ (estatura)

$\mathrm{EE}=-1,009+0,023$ (idade) - 0,074 (Escolaridade) 


\section{DISCUSSÃO}

A cidade de São Paulo permitiu obter uma amostra com indivíduos com características físicas diversas e pertencentes a vários grupos raciais.

A idade mínima de 21 anos foi estabelecida para a participação neste estudo, porque o alongamento do comprimento axial cessa ao redor da adolescência ${ }^{(20)} \mathrm{e}$ devido à necessidade de assinatura do consentimento livre do paciente.

As cirurgias oculares prévias ${ }^{(21-23)}$ e o diabetes ${ }^{(24)}$ podem interferir com os parâmetros biométricos. Assim, os indivíduos com esses antecedentes pessoais não foram incluídos na amostra.

A escolha da biometria ultra-sônica para medida dos parâmetros biométricos (Figura 2) foi realizada após avaliação das publicações comparando as técnicas e pela maior viabilidade na execução da coleta de dados. Alguns autores em 2003 encontraram alta correlação entre as medidas realizadas por aparelho de biometria ultra-sônica (Ultrascan ${ }^{\circledR}$ ) e de biometria óptica (IOL Master ${ }^{\circledR}$ ) e que não há diferença estatisticamente significante na acurácia das medidas quando comparada a técnica de contato e imersão, realizadas por examinador experiente ${ }^{(25)}$.

Em relação à idade, os indivíduos da presente amostra apresentaram uma distribuição ampla, sendo que a faixa etária mais representada foi a de 40 a 49 anos $(36,42 \%)$.

A média de escolaridade de $6,96 \pm 4,35$ anos foi semelhante à do CENSO $2000^{(17)}$, que foi de 6,2 anos, na avaliação de maiores de 15 anos.

Embora não tenha sido encontrada nos modelos de regressão múltipla uma correlação estatisticamente significante entre escolaridade, comprimento axial e profundidade da cavidade vítrea, que ratificaria a hipótese de que o esforço acomodativo levaria a um aumento do comprimento axial às custas de um aumento na profundidade da cavidade vítrea, como já relata$\mathrm{do}^{(26)}$, foi encontrada uma correlação negativa estatisticamente significante entre escolaridade e equivalente esférico.

A estatura média encontrada nos indivíduos deste estudo foi $160,26 \pm 8,23 \mathrm{~cm}$, semelhante à encontrada em adultos chineses da Singapura em que a estatura média encontrada foi $159 \pm 8 \mathrm{~cm}^{(27)}$. A maioria dos estudos realizados para avaliação da estatura e da tendência secular em estatura utiliza dados de indivíduos do sexo masculino ao apresentarem-se para o serviço militar ${ }^{(28-29)}$, impossibilitando a comparação a este estudo em que a maioria dos indivíduos foi do sexo feminino $(64,74 \%)$ e de diferentes idades.

\section{Parâmetros Biométricos}

Comprimento axial (CAx): O CAx mais longo nos indivíduos com maior estatura já foi relatado por outros $\operatorname{estudos}^{(10,30)}$.

Nos indivíduos mais jovens o achado de maior CAx ratifica o conceito da tendência secular em estatura ${ }^{(31)}$ e está de acordo com outros estudos populacionais ${ }^{(27,30,32)}$. O menor CAx nos mais idosos justifica a tendência à hipermetropia encontrada nestes indivíduos quando ainda não apresentam catarata.

O tendência a apresentar maior CAx os indivíduos do sexo masculino $(\mathrm{CAx}=23,19 \mathrm{~mm})$ do que no sexo feminino $(\mathrm{CAx}=$ $22,73 \mathrm{~mm}$ ), foi relatado em belgas ${ }^{(33)}$, mongolianos $^{(34)}$, da Arábia Saudita $^{(35)}$ e em crianças chinesas ${ }^{(36)}$, e pode ser relacionado à maior estatura normalmente encontrada nos homens. Entretanto, no modelo de regressão o sexo não pareceu interferir estatisticamente em nenhum dos parâmetros analisados.

$\mathrm{Na}$ aplicação do modelo de regressão linear apenas a estatura apresentou influência estatística no CAx.

Profundidade da câmara anterior (PCA): A diminuição PCA com a idade, pode estar relacionada ao menor CAx e ao aumento da EC nos indivíduos com maior idade. Achado este, que já foi alguns estudos ${ }^{(27,37-38)}$, assim como o aumento da PCA com a estatura ${ }^{(10)}$.

Espessura do cristalino (EC): Encontrou-se a EC relacionada apenas à idade, com aumento de espessura de $0,02 \mathrm{~mm} /$ ano $^{(39)}$, referente ao contínuo crescimento do cristalino ${ }^{(40)}$.

Profundidade da cavidade vítrea (PCV): A PCV apresentou-se positivamente relacionada à estatura e negativamente à idade, achado compatível com a literatura ${ }^{(10,27)}$.

Ceratometria média: Apresentou correlação negativa moderada com o CAx $(r=-0,59)$, relação esta que favorece o processo de emetropização ${ }^{(33)}$. No modelo de regressão a ceratometria média apresentou-se relacionada apenas à estatura.

Erro refrativo: Os míopes foram o menor grupo $(12,72 \%)$ e apresentaram tendência a CAx mais longo, PCA maior, EC menor, $\mathrm{PCV}$ maior, $\mathrm{K}$ mais plana, grau de escolaridade mais elevado, estatura e idade semelhantes aos emétropes, mas inferiores a dos hipermétropes.

No modelo de regressão linear, observou-se que apenas a idade e a escolaridade influenciaram o EE do erro refrativo.

Neste estudo, assim como em outro realizado em chineses encontrou-se que a estatura apresentou-se relacionada às dimensões oculares ${ }^{(27)}$ embora não influenciasse diretamente a refração. Ou seja, embora pessoas altas tenderiam a ter olhos mais longos, estes não seriam necessariamente míopes.

A maioria dos estudos publicados previamente mostra homens e mulheres considerados como uma amostra homogênea, e seus resultados válidos para ambos os sexos. Alguns já relataram diferenças nas medidas dos parâmetros oculares entre homens e mulheres ${ }^{(27,33-34,41)}$, sendo o comprimento axial no sexo feminino 0,4 a $0,8 \mathrm{~mm}$ menor que no sexo masculino ${ }^{(12)}$. A explicação para essa diferença não é clara, mas provavelmente refletiria as diferenças genéticas ${ }^{(1,12,42)}$.

Em mongolianos foi relatado que os homens tendiam a ter CAx mais longo e curvatura corneana menos curva que as mulheres da mesma idade. Entretanto, essa diferença não se mostrou significante após o controle da estatura ${ }^{(34)}$. Dessa forma, pode-se inferir que o mesmo poderia acontecer com a presente amostra.

Comentários, críticas e limitações: O presente estudo tem mérito por ter sido realizado em adultos brasileiros correlacionando as variáveis estudadas e permitindo a formulação de equações para o estabelecimento do valor de cada parâmetro biométrico, do erro refrativo e do equivalente esférico.

A utilização da demanda espontânea evitou os vieses dos 
estudos com grupos de indivíduos específicos e altamente selecionados (biomicroscopistas, estudantes de medicina, militares).

Este estudo populacional, assim como a maioria dos outros (chineses, esquimós, mongolianos, americanos), é transversal. Deste modo, as inferências longitudinais devem ser consideradas com extrema cautela. $\mathrm{O}$ tamanho da amostra não foi muito grande, portanto as conclusões com relação a esta amostra devem ser cuidadosas.

\section{CONCLUSÕES}

O comprimento axial apresentou-se positivamente relacionado à estatura. A profundidade da câmara anterior apresentou-se positivamente relacionada à estatura e negativamente relacionada à idade do indivíduo. A espessura do cristalino apresentou-se positivamente relacionada unicamente à idade. A profundidade da cavidade vítrea apresentou-se positivamente relacionada à estatura e negativamente à idade. A ceratometria média apresentou-se positivamente relacionada apenas à estatura. $\mathrm{O}$ equivalente esférico apresentou-se positivamente relacionado à idade e negativamente à escolaridade. $\mathrm{O}$ sexo não apresentou relação com os parâmetros analisados. Estes achados referentes à raça brasileira não diferem muito dos encontrados em outras populações, no que concerne aos parâmetros biométricos oculares.

\section{ABSTRACT}

Purpose: To assess ocular biometric parameters and refractive error in Brazilian adults and their relationship with height, age, gender and years of formal education. Methods: Crosssectional study that assessed 173 subjects by keratometry, echobiometry, refraction and measurement of body height. The statistical analysis was performed using Pearson's coefficient and a regression model was constructed. Results: The correlations found were: each $10 \mathrm{~cm}$ - increase in height was related to a $0.32 \mathrm{~mm}$ longer axial length, $0.07 \mathrm{~mm}$ deeper anterior chamber, $0.26 \mathrm{~mm}$ deeper vitreous chamber and $0.50 \mathrm{D}$ flatter keratometry. Each 10-year increase in age, related to a $0.15 \mathrm{~mm}$ smaller axial length, $0.25 \mathrm{~mm}$ thicker lens, $0.21 \mathrm{~mm}$ shallower vitreous chamber and $0.23 \mathrm{D}$ more positive spherical equivalent. Each 10-year increase in education related to a $0.74 \mathrm{D}$ more negative spherical equivalent. Gender did not influence the analyzed biometric parameters. Equations referring to biometric parameters and spherical equivalent were formulated. Conclusions: Positive correlations were found between: axial length and height; anterior chamber and vitreous cavity depth with height; lens thickness with age; keratometry with height; and spherical equivalent with age. Negative correlations were found between: anterior chamber and vitreous cavity depth with age; spherical equivalent and years of formal education.

Keywords: Eye; Biometry; Refractive errors; Body height; Age factors; Sex factors; Educational status

\section{REFERÊNCIAS}

1. Brown NP, Koretz JF, Bron AJ. The development and maintenance of emmetropia. Eye. 1999;13(Pt1):83-92.

2. Larsen JS. The sagittal growth of the eye. II. Ultrasonic measurement of the axial diameter of the lens and the anterior segment from birth to puberty. Acta Ophthalmologica. 1971;49(3):427-40.

3. McBrien NA, Adams DW. A longitudinal investigation of adult-onset and adult-progression of myopia in an occupational group. Refractive and biometric findings. Invest Ophthalmol Vis Sci. 1997;38(2):321-33.

4. Adams DW, McBrien NA. Prevalence of myopia and myopic progression in a population of clinical microscopists. Optometry \& Vision Science. 1992;69 (6):467-73.

5. Young FA. The nature and control of myopia. J Am Optom Assoc. 1977;48 (4):451-7.

6. Kinge B, Midelfart A, Jacobsen G, Rystad J. The influence of near-work on development of myopia among university students. A three-year longitudinal study among engineering students in Norway. Acta Ophthalmologica Scandinavica. 2000;78(1):26-9.

7. Avetisov ES, Savitskaya NF, Vinetskaya MI, Iomdina EN. A study of biochemical and biomechanical qualities of normal and myopic eye sclera in humans of different age groups. Metab Pediatr Syst Ophthalmol. 1983;7(4): 183-8.

8. Schaeffel F, Troilo D, Wallman J, Howland HC. Developing eyes that lack accommodation grow to compensate for imposed defocus. Vis Neurosci. 1990; 4(2):177-83.

9. Larsen JS. Axial length of the emmetropic eye and its relation to the head size. Acta Ophthalmol (Copenh). 1979;57(1):76-83.

10. Wong TY, Foster PJ, Johnson GJ, Klein BE, Seah SK. The relationship between ocular dimensions and refraction with adult stature: the Tanjong Pagar Survey. Invest Ophthalmol Vis Sci. 2001;42(6):1237-42.

11. Larsen JS. The sagittal growth of the eye. 1. Ultrasonic measurement of the depth of the angetior chamber from birth to puberty. Acta Ophthalmol (Copenh). 1971;49(2):239-62.

12. Midelfart A. Women and men-same eyes? Acta Ophthalmol Scand. 1996;74 (6):589-92.

13. Ninn-Pedersen K, Stenevi U, Ehinger B. Cataract patients in a defined Swedish population 1986-1990. I. Resources and epidemiology. Acta Ophthalmol (Copenh). 1994;72(1):1-9.

14. Lyhne N, Sjolie AK, Kyvik KO, Green A. The importance of genes and environment for ocular refraction and its determiners: a population based study among 20-45 year old twins. Br J Ophthalmol. 2001;85(12):1470-6.

15. Saw SM, Gazzard G, Au Eong KG, Tan DT. Myopia: attempts to arrest progression. Br J Ophthalmol. 2002;86(11):1306-11.

16. Fredrick DR. Myopia: was mother right about reading in the dark? $\mathrm{Br} \mathrm{J}$ Ophthalmol. 2001;85(5):509-10. Comment on: Br J Ophthalmol. 2001;85(5): 527-8.

17. Instituto Brasileiro de Geografia e Estatística. IBGE. Censo Demográfico 2000 Características gerais da população: resultados da amostra. Rio de Janeiro: Instituto Brasileiro de Geografia e Estatística; 2003.

18. Byrne SF. A-scan axial eye length measurements. Mars Hill: Grove Park; 1995.

19. Pinheiro RK, Netto AL. Estudo comparativo da acomodação residual após a instilação de colírios de tropicamida a $1 \%$, ciclopentolato a $1 \%$ e associação de tropicamida a 1\% + ciclopentolato a 1\%. Arq Bras Oftalmol. 2000;63(6): 475-9.

20. Sorsby A, Leary GA. A longitudinal study of refraction and its components during growth. Spec Rep Ser Med Res Counc (G B). 1969;309:1-41.

21. Kook MS, Kim HB, Lee SU. Short-term effect of mitomycin-C augmented trabeculectomy on axial length and corneal astigmatism. J Cataract Refract Surg. 2001;27(4):518-23.

22. Nemeth J, Horoczi Z. Changes in the ocular dimensions after trabeculectomy. International Ophthalmology. 1992;16(4-5):355-7.

23. Francis BA, Wang M, Lei H, Du LT, Minckler DS, Green RL, et al. Changes in axial length following trabeculectomy and glaucoma drainage surgery. Br J Ophthalmol. 2005;89(1):17-20.

24. Pierro L, Brancato R, Robino X, Lattanzio R, Jansen A, Calori G. Axial length in patients with diabetes. Retina. 1999;19(5):401-4.

25. Nemeth J, Fekete O, Pesztenlehrer N. Optical and ultrasound measurement of axial length and anterior chamber depth for intraocular lens power calculation. J Cataract Refract Surg. 2003;29(1):85-8. Comment in: J Cataract Refract Surg. 2003;29(1):1-3. 
26. Wong TY, Foster PJ, Johnson GJ, Seah SK. Education, socioeconomic status, and ocular dimensions in Chinese adults: the Tanjong Pagar Survey. Br J Ophthalmol. 2002;86(9):963-8.

27. Wong TY, Foster PJ, Ng TP, Tielsch JM, Johnson GJ, Seah SK. Variations in ocular biometry in an adult Chinese population in Singapore: the Tanjong Pagar Survey. Invest Ophthalmol Vis Sci. 2001;42(1):73-80.

28. Steckel R, Floud R. Health and welfare during industrialization. Chicago: Chicago University; 1997.

29. Marcondes E, Marques RM. Estudo antropométrico de indivíduos aptos para o serviço militar no período de 1979 a 1991. Rev Bras Crescimento Desenvolv Hum. 1993;3(2):9-38.

30. Johnson GJ, Matthews A, Perkins ES. Survey of ophthalmic conditions in a Labrador community. I. Refractive errors. Br J Ophthalmol. 1979;63(6):440-8.

31. França Junior I, Silva GR, Monteiro CA. Tendência secular da altura na idade adulta de crianças nascidas na cidade de São Paulo entre 1950 e 1976. Rev Saúde Pública. 2000;34(6 Supl):102-7.

32. Grosvenor T. Reduction in axial length with age: an emmetropizing mechanism for the adult eye? Am J Optom Physiol Opt. 1987;64(9):657-63.

33. Francois J, Goes F. Ultrasonographic study of 100 emmetropic eyes. Ophthalmologica. 1977; 175(6):321-7.

34. Wickremasinghe S, Foster PJ, Uranchimeg D, Lee PS, Devereux JG, Alsbirk
$\mathrm{PH}$, et al. Ocular biometry and refraction in Mongolian adults. Invest Ophthalmol Vis Sci. 2004;45(3):776-83.

35. Osuobeni EP. Ocular components values and their intercorrelations in Saudi Arabians. Ophthalmic Physiol Opt. 1999;19(6):489-97.

36. Saw SM, Gazzard G, Koh D, Farook M, Widjaja D, Lee J, et al. Prevalence rates of refractive errors in Sumatra, Indonesia. Invest Ophthalmol Vis Sci. 2002;43(10):3174-80.

37. Wojciechowski R, Congdon N, Anninger W, Teo Broman A. Age, gender, biometry, refractive error, and the anterior chamber angle among Alaskan Eskimos. Ophthalmology. 2003;110(2):365-75.

38. Alsbirk PH. Variation and heritability of ocular dimensions. A population study among adult Greenland Eskimos. Acta Ophthalmol (Copenh). 1977;55(3):443-56.

39. Brown NP. Changes in lens curvature with age. Exp Eye Res. 1974;19(2):175-83.

40. Cook CA, Koretz JF, Pfahni A, Hyun J, Kaufman PL. Aging of the human crystalline lens and anterior segment. Vision Res. 1994;34(22):2945-54.

41. Mutti DO, Mitchell GL, Moeschberger ML, Jones LA, Zadnik K. Parental myopia, near work, school achievement, and children's refractive error. Invest Ophthalmol Vis Sci. 2002;43(12):3633-40.

42. Mutti DO, Zadnik K, Adams AJ. Myopia. The nature versus nurture debate goes on. Invest Ophthalmol Vis Sci. 1996;37(6):952-7. Review. No abstract available. Comment in: Invest Ophthalmol Vis Sci. 1996;37(13):2533-4.



